\begin{tabular}{|c|l|}
\hline Title & Orientation and deformation of mineral crystals in tooth surfaces \\
\hline Author(s) & Fujisaki, Kazuhiro; Todoh, Masahiro; Niida, A tsushi; Shibuya, Ryota; Kitami, Shunsuke; Tadano, Shigeru \\
\hline Citation & $\begin{array}{l}\text { Journal of the Mechanical Behavior of Biomedical Materials, 10, 176-182 } \\
\text { https:/doi.org/10.1016/.jmbbm.2012.02.025 }\end{array}$ \\
\hline Issue Date & 2012-06 \\
\hline Doc URL & http://hdl.handle.net/2115/49714 \\
\hline Rights & Copyright Elsevier \\
\hline Type & article (author version) \\
\hline File Information & jmbbm10_176-182.pdf \\
\hline
\end{tabular}

Instructions for use 


\title{
Orientation and Deformation of Mineral Crystals in Tooth Surfaces
}

\author{
Kazuhiro Fujisaki ${ }^{\mathrm{a}}$, Masahiro Todoh ${ }^{\mathrm{a}}$, Atsushi Niida ${ }^{\mathrm{b}}$, Ryota Shibuya ${ }^{\mathrm{a}}$, \\ Shunsuke Kitami ${ }^{\mathrm{a}}$, Shigeru Tadano ${ }^{\mathrm{a}}$ \\ ${ }^{a}$ Division of Human Mechanical Systems and Design, Faculty of Engineering, \\ Hokkaido University, Kita-13, Nishi-8, Kita-ku, Sapporo, Hokkaido 060-8628, JAPAN \\ ${ }^{\mathrm{b}}$ Health Science University of Hokkaido, Kanazawa, Tobetsu-cho, Ishikari-gun, \\ Hokkaido 060-0293, JAPAN
}

\section{Corresponding Author:}

Kazuhiro Fujisaki (Ph.D.)

Faculty of Engineering, Hokkaido University, Kita 13, Nishi 8, Kita-ku, Sapporo 060-8628, JAPAN

Email: fujiwax@eng.hokudai.ac.jp

Tel \& Fax: +81-11-706-6396 


\begin{abstract}
Tooth enamel is the hardest material in the human body, and it is mainly composed of hydroxyapatite (HAp)-like mineral particles. As HAp has a hexagonal crystal structure, X-ray diffraction methods can be used to analyze the crystal structure of HAp in teeth. Here, the X-ray diffraction method was applied to the surface of tooth enamel to measure the orientation and strain of the HAp crystals. The c-axis of the hexagonal crystal structure of HAp was oriented to the surface perpendicular to the tooth enamel covering the tooth surface. Thus, the strain of HAp at the surface of teeth was measured by X-ray diffraction from the (004) lattice planes aligned along the c-axis. The X-ray strain measurements were conducted on tooth specimens with intact surfaces under loading. Highly accurate strain measurements of the surface of tooth specimens were performed by precise positioning of the X-ray irradiation area during loading. The strains of the (004) lattice plane were measured at several positions on the surface of the specimens under compression along the tooth axis. The strains were obtained as tensile strains at the labial side of incisor tooth specimens. In posterior teeth, the strains were different at different measurement positions, varying from tensile to compressive types.
\end{abstract}

Key words: Biomechanics, Tooth enamel, Hydroxyapatite, X-ray diffraction, Crystal orientation, Strain 


\section{Introduction}

Teeth have a layered structure formed by tooth enamel that covers the dentin containing tooth pulp. The tooth enamel is the hardest and most highly mineralized tissue in the human body. The mechanical properties of tooth enamel are well investigated and described as anisotropic elastic owing to its hierarchical structure and nanostructural behavior (He and Swain, 2008). The microscopic tissue structure of tooth enamel is organized into numerous enamel prisms with column-shaped structures and cavities. The tissue is subject to an uneven stress distribution with localized stress concentrations because of the boundary of the enamel-dentin junction (EDJ), micro-scale cavities, and unusual force transfer in the tissue due to biting hard food and poor matching of the biting surfaces of teeth. These stress concentrations generate cracks that may cause the tooth to fracture. Stress analyses of tooth structure have been conducted using numerical simulation techniques such as the finite element method. Rodrigues et al. (2009) showed the complex stress distribution on the tooth surface due to compression during biting. Miura et al. (2009) indicated the presence of microscopic stress concentrations generated by differences in the microstructure of enamel rods. The measurement of stresses and strains in tooth enamel is expected to be of use in the estimation of tooth stiffness, evaluation of dental occlusion, and assessment of compatibility of implant replacements. As hydroxyapatite (HAp)-like mineral material has a hexagonal crystal structure, X-ray diffraction can be used to analyze its micro/nano-scale structure (Nakano et al., 2002; Fujisaki et al., 2006, 2007; Giri et al., 2009). The HAp crystal has an anisotropic elastic modulus, as determined by a technique based on elastic wave propagation (Gardner et al., 1992) and by nano-indentation measurements (Zamiri and De, 2011). The mechanical properties of macroscopic enamel tissue are strongly dependent on the alignment and orientation of the HAp crystals, as found from elastic modulus (Habelitz et al., 2001) and fatigue toughness (Bajaj et al., 2008) measurements. The strain behavior of HAp in mineralized tissue has been extensively studied using synchrotron X-ray sources that generate high-brightness X-rays for bone tissue (Almer and Stock, 2005, 2007; 
Gupta et al., 2006a, 2006b) and for tooth dentin and tooth enamel (Almer and Stock, 2010). These studies have focused on the strains in the internal structure of tissue under loading of thin specimens or the intact structure of an isolated tooth, measured by transmitting X-rays through the entire tissue. The tooth surfaces formed by tooth enamel are required to have high abrasion resistance and high corrosion resistance. The changes in the surface structure caused by caries or microscopic damage may lead to significant impairment of the normal functions of teeth, and eventually, loss of teeth. The HAp crystal structure is altered by tooth decay in the early stage of caries, as suggested by some studies (Yagi et al., 2009; Tanaka et al., 2010). The stress/strain distributions are important indicators of the stiffness of teeth as force bearing tissue during actual biting. The strain on the tooth should preferably be investigated under in vivo conditions. In this study, we used the X-ray diffraction method to measure the strain of the tooth surface with low X-ray intensities. The measurement system was improved to apply the method to shallow areas of the tooth enamel that have surface irregularities and/or that are subject to surface-level changes during loading. The requirements for precision measurements by X-ray diffraction in these types of surfaces and the limitations of the diffraction method were investigated using human tooth specimens.

\section{Method}

The X-ray diffraction method is used to analyze the periodic structure of molecules, atoms, and crystal lattice planes. When characteristic X-rays with a unique wavelength $\lambda$ are irradiated onto the surface of tooth enamel, the X-rays are diffracted from the apatite crystal lattice; the angle of diffraction is $2 \theta$, with respect to the axis of the irradiated X-rays. This angle is determined by the interplanar spacing $d$ between the lattice planes of the crystals, according to Bragg's law, i.e., $2 d \sin \theta=\mathrm{n} \lambda$. Diffracted X-rays are generated from each layer of the crystal lattice planes that may have different interplanar spacings. When the crystal lattices are 
deformed by a strain $\varepsilon$, the angle $\theta_{0}$ changes to $\theta$ according to the interplanar spacing. Thus, $\varepsilon$ is obtained by measuring these angles, as shown in Eq. (1).

$$
\varepsilon=\frac{d-d_{0}}{d_{0}}=\frac{\sin \theta_{0}-\sin \theta}{\sin \theta}
$$

The measurable strain of the crystal lattice corresponds to the nominal axis of X-ray diffraction. Therefore, the strain component perpendicular to the tooth surface is obtained in the present study.

When the X-rays are used to irradiate materials consisting of one type of crystal or specific regions of crystals that are uniformly aligned in one direction, the diffracted X-rays concentrate in one direction showing a high X-ray intensity. The degree of orientation is generally evaluated by the intensity of the diffracted X-rays compared with the intensities measured in powders or polycrystalline materials with random orientations of crystal patterns. The orientation is well evaluated using the parameter $<\cos ^{2} \beta>$ calculated from only the diffracted X-ray intensity distribution on the Debye ring (Giri et al. 2009). The parameter is calculated as shown in Eq. (2),

$$
<\cos ^{2} \beta>=\frac{\int_{0}^{\pi} I_{(\beta)} \cos ^{2} \beta \sin \beta \cdot d \beta}{\int_{0}^{\pi} I_{(\beta)} \sin \beta \cdot d \beta}
$$

where $\beta$ is the azimuthal angle with respect to the $\mathrm{X}$-ray irradiation axis. The angles were measured with respect to the major orientation of the c-axis $(\beta=0)$, which was determined by intensity distribution analysis on the Debye ring.

X-ray pole figure measurements have been used to observe the orientation of crystal grains and their distributions in a polycrystalline material. This method can determine the major orientation of specific lattice planes via inclined X-ray irradiation on a material surface. This analysis has been used to determine the specific axis of the HAp crystal structure in mineralized 
tissue (Sasaki and Sudoh, 1997). The HAp crystal orientations at tooth surfaces were obtained in the present study.

X-ray intensity logarithmically decreases with an increase in the depth of the irradiated material. The ratio of output to input X-ray intensity transmitted over a distance $x$ is described as $I_{\text {out }} / I_{\text {in }}=\exp [-\mu x]$. The X-ray absorption coefficient $\mu$ of HAp, regarded to be almost equal to that of tooth enamel tissue, was calculated to be $29.4 \mathrm{~cm}^{-1}$ by considering the absorption ability of each constituent atom of HAp using Mo-K $\alpha$ X-rays with a wavelength of $0.071 \mathrm{~nm}$ (Todoh et al., 2009). For Co-K $\alpha$ X-rays with a wavelength of $0.179 \mathrm{~nm}$, the coefficient was $396.3 \mathrm{~cm}^{-1}$, calculated in the same manner. In X-ray diffraction studies, the X-ray penetration path is determined from the Bragg angle $\theta$. When the nominal axis of diffraction is perpendicular to the specimen surface, the penetration path length of the X-rays reaches a depth $z$, i.e., $2 z / \sin \theta$. Then, the intensity ratio of X-rays diffracted from the region with depth $z, I$, to the surface without X-ray absorption, $I_{0}$, is given by Eq. (3) (Fujisaki and Tadano, 2010).

$$
\frac{I}{I_{0}}=\exp \left[-\mu \frac{2 z}{\sin \theta}\right]
$$

The changes in the intensity of X-rays diffracted from (002) and (004) lattice planes with different Bragg angles corresponding to the c-axis of HAp crystals at depth $z$ are shown in Fig. 1. The X-ray diffracted from $0.1-\mathrm{mm}$ depth shows approximately $5 \%$ of the X-ray intensity at the surface $(z=0)$ in the case of Mo-K $\alpha$ rays. For Co-K $\alpha$ rays, only a $0.02-\mathrm{mm}$ depth is required to reduce the intensity to less than $5 \%$ of the X-ray intensity at the surface.

\section{Experimental Procedure}

\subsection{Materials}

Specimens were prepared from human teeth preserved in dry conditions after extraction 
during clinical treatment for dental implant replacement. Each specimen was free of decay and none had any significant diseases. Three types of specimens were prepared in the present study. A sliced specimen (1-mm thick) was cut out from a posterior tooth, which was used for the confirmation of crystal orientations in the tooth enamel. The slice was cut in the middle of the tooth, and the slicing plane was aligned with the lingual to the buccal side of the tooth. An intact posterior tooth was used for the pole figure measurements of the surface. Compression specimens formed by excision of both the top of the tooth crown and the bottom of the tooth root to ensure parallel surfaces were prepared from an incisor tooth and a posterior tooth. A strain gauge (KFG-1N-120 C1-11L3M3R, Kyowa Electronic Instruments Co., Ltd.) was attached to the side surface of each specimen to measure the macroscopic strain aligned with the tooth axis and corresponding to the loading direction.

\subsection{X-ray diffraction system}

The X-ray pole figure measurements of the crystal orientation were conducted using an X-ray diffractometer (RINT2200, Rigaku Corp.) with a multi-axial stage (MPA-2000, Rigaku Corp.). The pole figure measurements for the HAp crystals on the surface of the tooth enamel were carried out along the c-axis of the hexagonal crystals and were conducted by detecting the diffracted X-rays from the (004) lattice planes. The axis of orientation and distribution of the c-axis orientations in the X-ray irradiated area was recorded in the form of a contour map, as shown in Fig. 2. The pole figure was measured using the characteristic X-rays of Co-K $\alpha$, which have limited penetration ability. The crystal orientations of HAp in the sections of tooth enamel were determined by measuring the transmission characteristics of Mo-K $\alpha$ X-rays with a very short wavelength. The diffracted X-rays were detected using a two-dimensional X-ray imaging plate (IP) (BAS-IP SR 127, 127×127 mm, FUJIFILM Corp.) and an IP reader (R-AXIS DS3C, Rigaku Corp.). The X-ray diffraction patterns were obtained as circular distributions of Debye rings, as shown in Fig. 3. The two-dimensional distribution of the crystal orientations of HAp in 
a plane almost perpendicular to the X-ray irradiation direction has been measured from the X-ray intensity distribution on a Debye ring (Tadano et al., 2008).

\subsection{X-ray diffraction profiles of tooth enamel}

Figure 4 shows an intensity-angle profile for the diffracted Mo-K $\alpha$ X-rays measured from the tooth enamel surface; the X-rays were generated at a tube power of $40 \mathrm{kV}$ and tube current of $40 \mathrm{~mA}$. The X-ray irradiation spot size was $1 \mathrm{~mm}$ in diameter, as determined by a collimator. Individual peaks representing the (002), (211), (213), and (004) lattice planes of the HAp crystals are clearly observed in the X-ray diffraction profile of the HAp crystals.

\subsection{Experimental setup for strain measurements}

For the strain measurements, the specimens were set in a loading device that can apply a constant compression load during X-ray irradiation. The loading device was placed in an X-ray diffractometer (Ultima 4, Rigaku Corp.) for the strain measurement. In X-ray diffraction measurements, the points of incidence of X-rays on the specimen surface must remain constant during a loading experiment. Shifts in the diffracted X-ray path due to movement of the specimen surface result in errors in the measurement of the diffraction angle. To prevent such movement, the loading device was placed on a height-adjustable stage. A laser displacement meter (LC-2400 series, Keyence Corp.) was used to adjust the irradiation point that changed because of the deformation of both the specimen and the loading device. The laser light from the displacement meter and the X-ray irradiation position was set at one position on the specimen to prevent errors due to the movement of the specimen. Figure 5 shows the X-ray diffraction profiles of the tooth surface, measured at $30-\mu \mathrm{m}$ intervals from $z=0$ to $150 \mu \mathrm{m}$ below this depth without loading. The results indicate that there was a profile shift without the application of the lattice strain. The amount of peak shift corresponded to a strain range of 0.15\% (1500 microstrain), calculated from Eq. (1). The position error should be reduced for 
providing precise measurements, and it was modified to be below $5 \mu \mathrm{m}(<0.005 \%=50$ microstrain) during this X-ray measurement. Characteristic X-rays of Mo-K $\alpha$ generated at the tube voltage of $40 \mathrm{kV}$ and tube current of $40 \mathrm{~mA}$ in the X-ray diffractometer were used in this experiment. The strain measurements were conducted under applied strains of $0.2 \%$ (2000 microstrain), measured using a strain gauge bonded to the surface of the specimen. The strains of the (004) lattice planes were measured at several positions on the surface of the tooth specimens during loading.

\section{Results}

\subsection{Crystal orientation}

The c-axis orientation on the surface of the tooth enamel was investigated from both pole figure measurements of the diffracted X-rays recorded on the reflection side and IP measurements on the X-ray penetration side. The three-dimensional alignment of specific lattice planes was expressed as a projected image of X-ray intensity distribution in a contour map obtained from pole figure measurements. Figure 6 shows the pole figures of the c-axis measured from the (004) lattice planes of the HAp crystals at several points on the posterior tooth surface. The highest peak appeared in the vicinity of the center of the pole figure measured in the flat regions A, B, E, and F of the specimen, as shown in Fig. 6. Two independent peaks appear in the pole figure of the concave and convex regions $\mathrm{C}$ and $\mathrm{D}$. These results indicate that the HAp crystals were aligned with the c-axis of the crystals that was oriented perpendicular to the tooth surface. Figure 7 shows the c-axis orientations in a section of the posterior tooth enamel. The HAp crystal orientations radiated outward from the EDJ to the tooth surface. The c-axis orientations were very similar to the longitudinal direction of the enamel prisms. Figure 8 shows the angle between the c-axis of the HAp crystal and the tooth axis (a), and the direction perpendicular to the outer surface (b). The degree of orientation of each measured region was 
represented by $\left\langle\cos ^{2} \beta\right\rangle$. Figure 8 (c) shows the distribution of $\left\langle\cos ^{2} \beta>\right.$. Regions with a strong degree of orientation were observed clearly in the thick enamel regions at the crown of the teeth. Overall, this shows that the (004) planes along the c-axis can be detected by surface observations of the tooth enamel by X-ray diffraction. This leads to the conclusion that lattice plane measurements may be used to determine the strain on the tooth surface.

\subsection{Strain measurement}

The (002) and (004) lattice planes along the c-axis of the HAp crystals were shown to be clearly oriented perpendicular to the surface of the tooth specimens. The peak profile of X-rays diffracted from the (004) lattice plane was used in the measurements. The strains can be measured in the direction perpendicular to compressive loading on the labial side. The strain value was regarded as the mean of that for the X-ray irradiation area and the X-ray penetration area. The X-ray penetration area can be determined from the data shown in Fig. 1. Although the detected X-ray intensity was determined by integrating the data from the surface to depth areas, the intensity was scarcely affected by X-rays generated from more than $0.1 \mathrm{~mm}$ deep area. Figure 9 shows the lattice strain values measured under applied strains of 2000 microstrain for compression along the tooth axis. The strains in the incisor tooth specimen were measured as tensile 670 microstrain at the vicinal location and 150 microstrain $4 \mathrm{~mm}$ away from the strain gauge. In the case of the posterior tooth specimen, the strain values differed depending on the measurement position, varying from tensile 520 microstrain to compressive 540 microstrain under the same applied load.

\section{Discussion}

This study applied the X-ray diffraction method to measure the crystal orientation of HAp in the surface of tooth enamel. The c-axis orientation represented by the alignment of the (004) 
lattice plane of the hexagonal HAp crystal structure was almost perpendicular to the surface. Al-Jawada et al. (2007) investigated the crystal orientation of HAp in a section of enamel using synchrotron X-rays and reported that the (002) lattice planes of the HAp crystals showed an acute angle with respect to the EDJ as compared to the c-axis. Simmons et al. (2011) investigated the c-axis orientations in tooth enamel with high resolution and discussed the three-dimensional inclination angles of these orientations. Although the alignment of the (004) lattice plane having the same direction as the (002) plane shown in the present study had a mean tendency of $>1 \mathrm{~mm}$ area, the presence of these orientations measured in the intact surface (Fig. 6) was coincident with the results.

The accuracy of X-ray strain measurements of tooth enamel decreases with movement of the surface. In this X-ray diffraction measurement, the errors in the strain values were $0.01 \%$ (100 microstrain) when the X-ray irradiation surface moved by just $10 \mu \mathrm{m}$. Thus, accurate positioning within a few micrometers is important for X-ray strain measurements on tooth surfaces. The shifts in the peaks of X-ray diffraction profiles with strain increase with the diffraction angle, according to Eq. (1). Using the (004) peak gives better results than the (002) peak because the diffraction angle of the (004) lattice plane is larger than that of the (002) lattice plane. This study performed X-ray diffraction measurements with errors less than $0.005 \%$ (50 microstrain) using a laser displacement meter. X-ray diffraction analysis can be used to perform quantitative measurements of both the crystal orientation and the lattice strain on the tooth surface.

The tooth specimens used in the present study were in a dry condition, which is different from in vivo conditions. Micro-sized cracks were often observed in the sectioned surfaces, particularly at the EDJ. The stress conditions in the compression loading experiments reported here are different from the actual conditions while biting. Although the compression load was regarded as uniaxial and uniformly distributed in the cross-sections of the specimens, uniform compression loading was difficult to apply to tooth specimens because the specimen shapes 
with excised top and bottom could not be prepared to have perfectly parallel surfaces. Further, the load distribution on the surface of teeth is not constant. The strains on the surface of the posterior tooth have been shown to have a complex distribution by means of finite element analysis (Rodrigues et al., 2009). The strains on the posterior tooth specimen showed different values and complex distributions even when measured at almost the same locations. These strain distributions on the surface of tooth enamel cause cracks and excessive abrasion of the surfaces. The X-ray diffraction method was used to perform strain distribution measurements using oriented HAp crystals in the enamel on the tooth surface. Although these measurements gave results only for the oriented crystals, this method can be used to estimate the strain of tooth surfaces with high resolution in comparison with other measurement techniques such as strain gauges. A micro-focused X-ray irradiation technique that employs a spot size less than 1-mm can provide higher resolution for strain measurements. This X-ray strain measurement technique is expected to be used in clinical applications for nondestructive and noncontact measurements.

\section{Conclusion}

The X-ray diffraction method was used to measure the strain of HAp crystals on the tooth enamel surface with high accuracy. The c-axis of the HAp crystals was aligned perpendicular to the surface of the tooth enamel. The (004) lattice plane could be used for strain measurements of the tooth surface. The strains were obtained as tensile strains perpendicular to the labial side surface of the incisor tooth specimen under compression along the tooth axis. In the posterior tooth specimen, the strain values differed depending on the measurement positions, varying from tensile to compressive under the same applied load. 


\section{Acknowledgement}

This work was supported by KAKENHI, Grants-in-Aid for Young Scientists (B) (22700461) and Scientific Research (A) (19200035).

\section{References}

Al-Jawad, M., Steuwer, A., Kilcoyne, S.H., Shore, R.C., Cywinski, R., Wood, D.J., 2007. 2D mapping of texture and lattice parameters of dental enamel. Biomaterials 28(18), 2908-2914.

Almer, J.D., Stock, S.R., 2005. Internal strains and stresses measured in cortical bone via high-energy X-ray diffraction. J. Struct. Biol. 152(1), 14-27.

Almer, J.D., Stock, S.R., 2007. Micromechanical response of mineral and collagen phases in bone. J. Struct. Biol. 157(2), 365-370.

Almer, J.D., Stock, S.R., 2010. High energy X-ray scattering quantification of in situ-loading-related strain gradients spanning the dentinoenamel junction (DEJ) in bovine tooth specimens. J. Biomech. 43(12), 2294-2300.

Bajaj, D., Nazari, A., Eidelman, N., Arola, D.D., 2008. A comparison of fatigue crack growth in human enamel and hydroxyapatite. Biomaterials 29, 4847-4854.

Fujisaki, K., Tadano, S., Sasaki, N., 2006. A method on strain measurement of HAP in cortical bone from diffusive profile of X-ray diffraction. J. Biomech. 39(3), 579-586.

Fujisaki, K., Tadano, S., 2007. Relationship between bone tissue strain and lattice strain of HAp crystals in bovine cortical bone under tensile loading. J. Biomech. 40(8), 1832-1838.

Fujisaki, K., Tadano, S., 2010. Strain measurement of pure titanium covered with soft tissue using X-ray diffraction. J. Biomech. Eng. 132(3), 0310041-0310045.

Gardner, T.N., Elliott, J.C., Sklar, Z., Briggs, G.A.D., 1992. Acoustic microscope study of the 
elastic properties of fluorapatite and hydroxyapatite, tooth enamel and bone. J. Biomech. 25(11), 1265-1277.

Giri, B., Tadano, S., Fujisaki, K., Sasaki, N., 2009. Deformation of mineral crystals in cortical bone depending on structural anisotropy. Bone 44, 1111-1120.

Gupta, H.S., Seto, J., Wagermaier, W., Zaslansky, P., Boesecke, P., Fratzl, P., 2006. Cooperative deformation of mineral and collagen in bone at the nanoscale. Proc. Natl. Acad. Sci. 103, $17741-17746$.

Gupta, H.S., Wagermaier, W., Zickler, G.A., Hartmann, J., Funari, S.S., Roschger, P., Wagner, H.D., Fratzl, P., 2006. Fibrillar level fracture in bone beyond the yield point. Int. J. Fract. 139, $425-436$.

Habelitz, S., Marshall, S.J., Marshall, G.W.Jr, Balooch, M., 2001. Mechanical properties of human dental enamel on the nanometre scale. Arch. Oral Biol. 46(2), 173-183.

He, L.H., Swain, M.V., 2008. Understanding the mechanical behaviour of human enamel from its structural and compositional characteristics. J. Mech. Behav. Biomed. 1(1), 18-29.

Miura, J., Maeda, Y., Nakai, H., Zako, M., 2009. Multi-scale analysis of stress distribution in teeth under applied forces. Dent. Mater. 25, 67-73.

Nakano, T., Kaibara, K., Tabata, Y., Nagata, N., Enomoto, S., Marukawa, E., Umakoshi, Y., 2002. Unique alignment and texture of biological apatite crystallites in typical calcified tissues analyzed by microbeam X-ray diffractometer system. Bone. 31(4), 479-487.

Rodrigues, F.P., Li, J., Silikas, N., Ballester, R.Y., Watts, D.C., 2009. Sequential software processing of micro-XCT dental-images for 3D-FE analysis. Dent. Mater. 25(6), e47-e55.

Sasaki, N., Sudoh, Y., 1997. X-ray pole figure analysis of apatite crystals and collagen molecules in bone. Calcif. Tissue Int. 60, 361-367.

Simmons, L.M., Al-Jawad, M., Kilcoyne, S.H., Wood, D.J., 2011. Distribution of enamel crystallite orientation through an entire tooth crown studied using synchrotron X-ray diffraction. Eur. J. Oral. Sci. 119 (Suppl. 1), 19-24. 
Tadano, S., Giri, B., Sato, T., Fujisaki, K., Todoh, M., 2008. Estimating nanoscale deformation in bone by X-ray diffraction imaging method. J. Biomech. 41(5), 945-952.

Tanaka, T., Yagi, N., Ohta, T., Matsuo, Y., Terada, H., Kamasaka, K., To-o, K., Kometani, T., Kuriki, T., 2010. Evaluation of the distribution and orientation of remineralized enamel crystallites in subsurface lesions by X-ray diffraction. Caries Res. 44(3), 253-259.

Todoh, M., Tadano, S., Giri, B., Nishimoto, M., 2009. Effect of gradual demineralization on the mineral fraction and mechanical properties of cortical bone. J. Biomech. Sci. Eng. 4(2), $230-238$.

Yagi, N., Ohta, N., Matsuo, T., Tanaka, T., Terada, Y., Kamasaka, H., To-o, K., Kometani, T., Kuriki, T., 2009. Evaluation of enamel crystallites in subsurface lesion by microbeam X-ray diffraction. J. Synchrotron Rad. 16(3), 398-404.

Zamiri, A., De, S., 2011. Mechanical properties of hydroxyapatite single crystals from nanoindentation data. J. Mech. Behav. Biomed. 4(2), 146-152. 


\section{Figures}

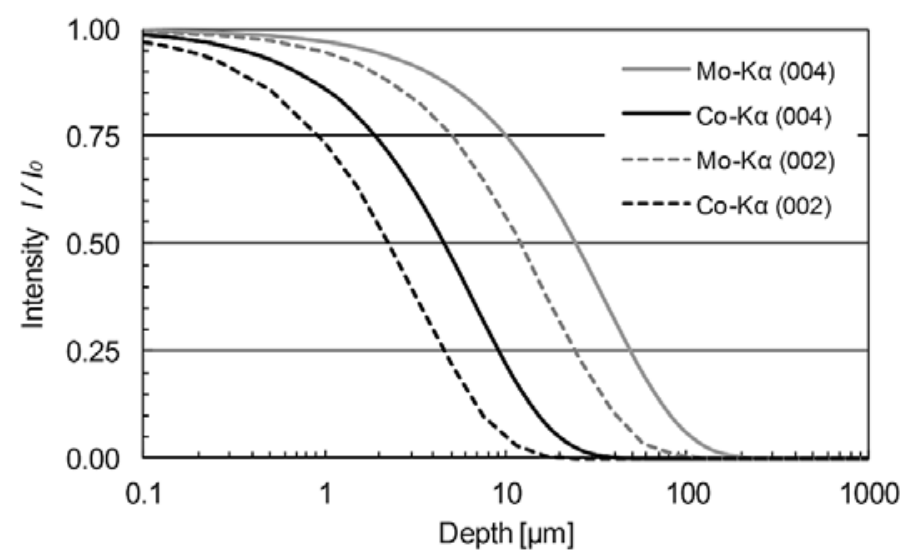

Fig. 1 Intensity of X-rays diffracted from deeper areas with long penetration path, $I$, calculated from Bragg angle, absorption coefficient of hydroxyapatite depending on type of characteristic X-rays (Mo-K $\alpha$ and Co- K $\alpha$ ), normalized by X-rays diffracted from surface, $I_{0}(\mathrm{depth}=0)$

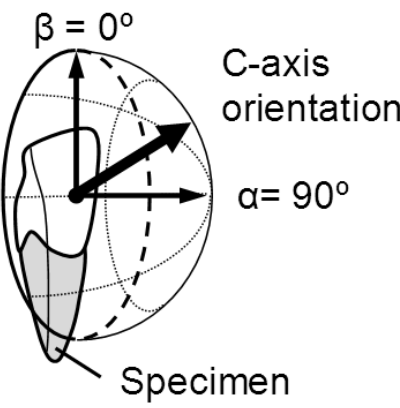

(a) Side view
X-ray intensity distribution

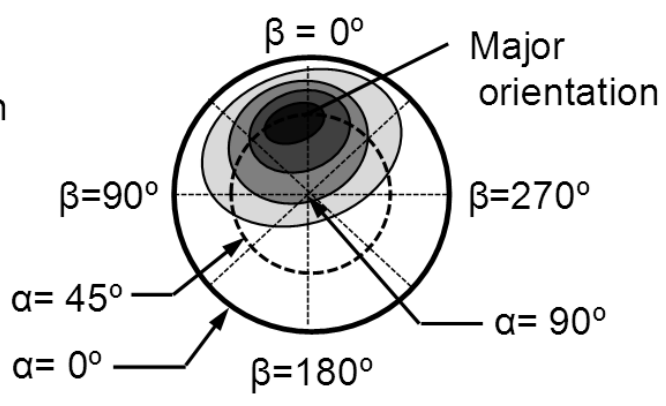

(b) Frontal view

Fig. 2 Geometry of X-ray pole figure measurements and crystal orientation axis on the surface of tooth specimens, (a) crystal orientation at X-ray irradiation position, (b) two-dimensional projection image of crystal orientation distributions 


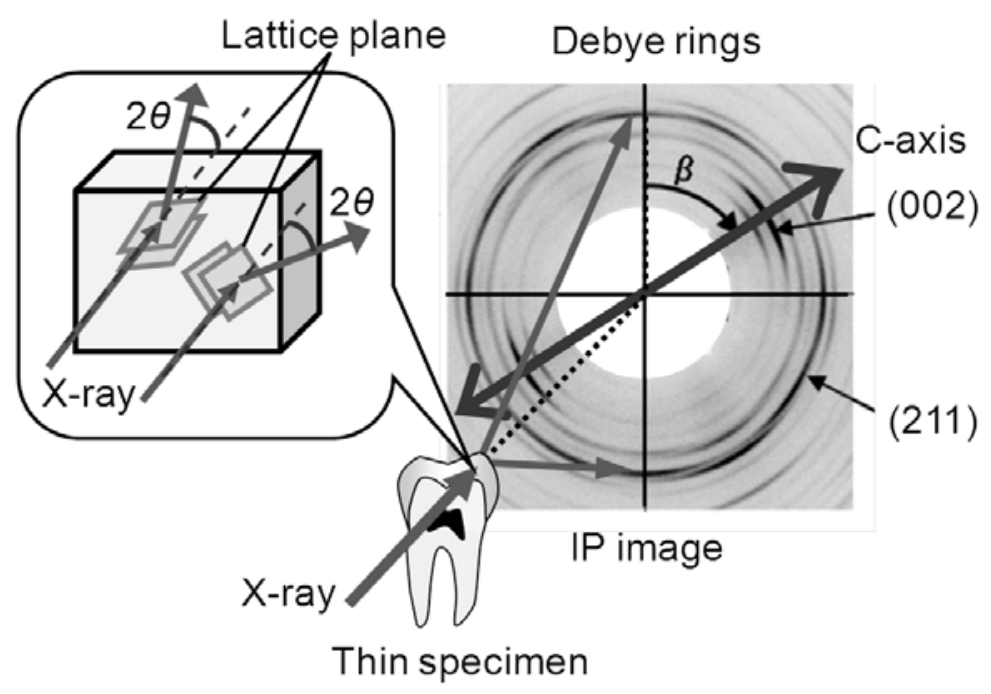

Fig. 3 Diffracted X-ray paths through tooth specimen and two-dimensional distribution patterns;

Debye rings, measured from tooth enamel

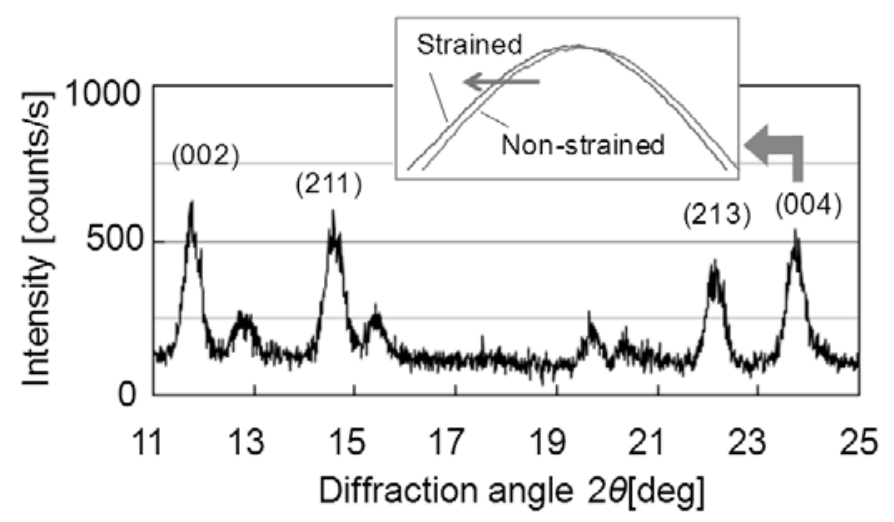

Fig. 4 X-ray diffraction profile of hydroxyapatite on the surface of tooth enamel; the profile is

shifted by the strain of the tooth 


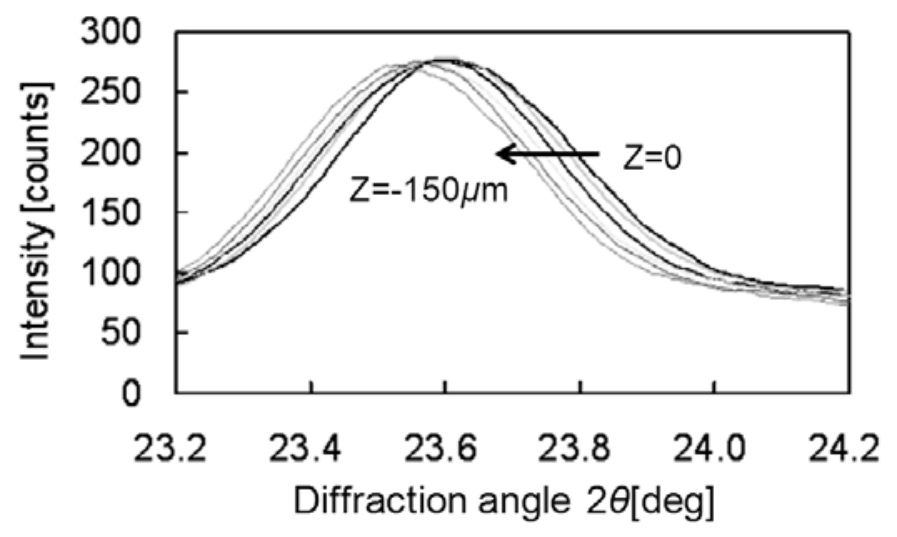

Fig. 5 Peak for the (004) lattice plane on X-ray diffraction profile shifted by a change in specimen position from 0 to $150 \mu \mathrm{m}$ without loading
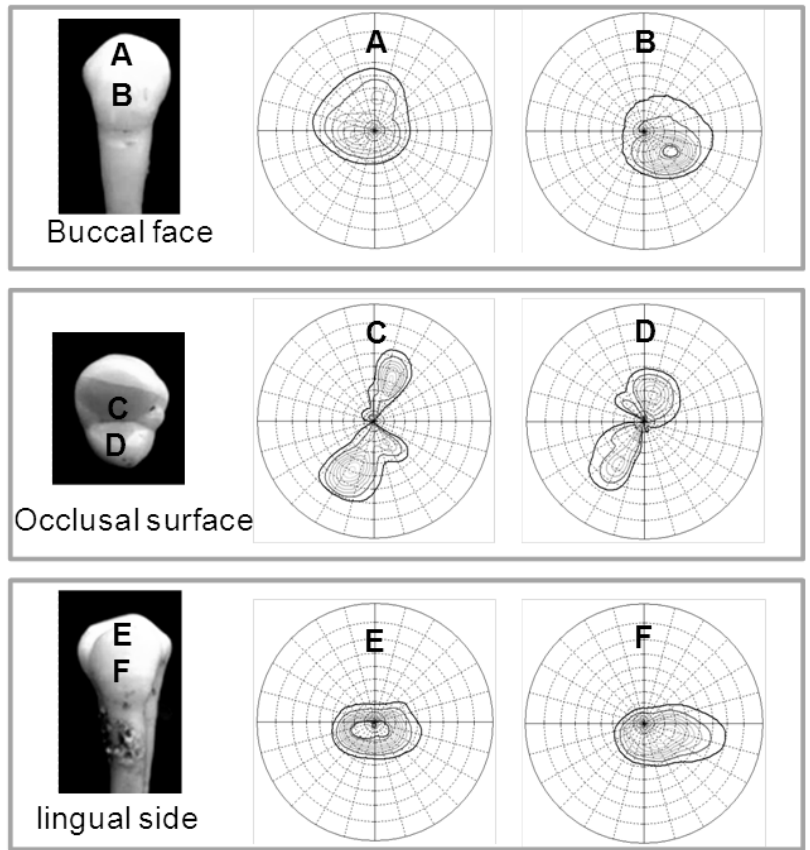

Fig. 6 Crystal orientation of c-axis of hydroxyapatite on the surface of tooth enamel of posterior tooth, as observed in X-ray pole figure measurements 


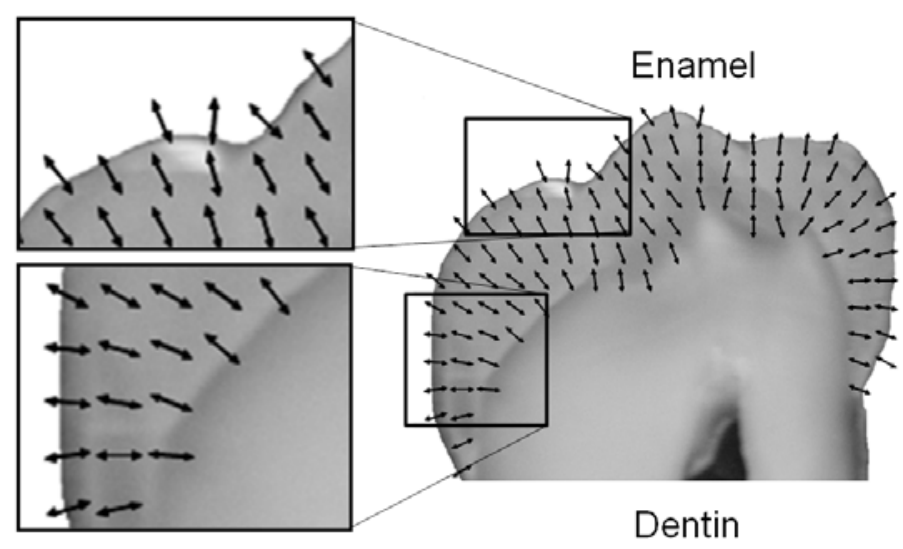

Fig. 7 Crystal orientation of hydroxyapatite with c-axis aligned perpendicular to surface of tooth enamel

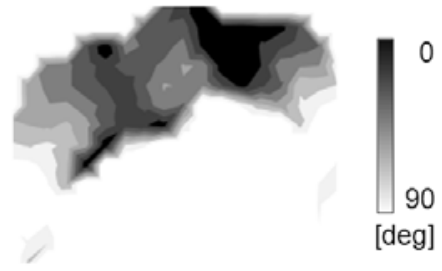

(a) Angle from tooth axis

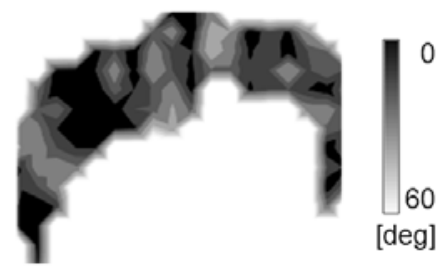

(b) Angle from surface perpendicular

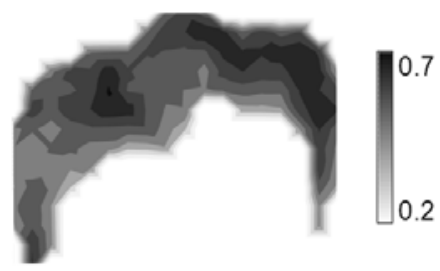

(c) Degree of orientation

Fig. 8 Characteristics of crystal orientation of hydroxyapatite c-axis in tooth enamel, (a) angles

between crystal orientation and tooth axes, (b) angles between crystal orientation axis and direction perpendicular to surface, (c) degree of orientation $\left\langle\cos ^{2} \beta>\right.$ calculated from major orientation at each measurement position, as shown in Fig. 7. 


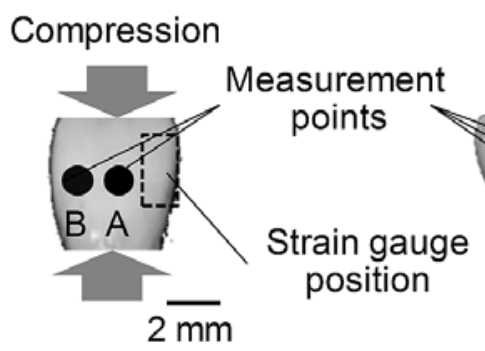
A: 673 [microstrain]
B: 150
R: 516
Incisor tooth
L: 90
T:-540
Posterior tooth

Fig. 9 Lattice strains perpendicular to surfaces of tooth specimens under uniaxial compression of 2000 microstrain $(0.2 \%)$ along tooth axis, measured using strain gauge. 